\title{
Navigation in the Presence of Humans*
}

\author{
E. A. Sisbot, R. Alami and T. Simeon \\ Robotics and Artificial Intelligence Group \\ LAAS/CNRS \\ 7, Avenue du Colonel Roche, 31077 Toulouse cedex 4 \\ France \\ \{easisbot, rachid,nic\}@laas.fr
}

\author{
K. Dautenhahn, M. Walters, S. Woods, \\ K. L. Koay and C. Nehaniv \\ University of Hertfordshire \\ College Lane, Hatfield Herts AL10 9AB \\ United Kingdom \\ K.Dautenhahn@herts.ac.uk
}

\begin{abstract}
Robot navigation in the presence of humans raises new issues for motion planning and control since the humans safety and comfort must be taken explicitly into account.

We claim that a human-aware motion planner must not only elaborate safe robot paths, but also plan good, socially acceptable and legible paths. Our aim is to build a planner that takes explicitly into account the human partner by reasoning about his accessibility, his vision field and potential shared motions.

This paper focuses on a navigation planner that takes into account the humans existence explicitly. This planner is part of a human-aware motion and manipulation planning and control system that we aim to develop in order to achieve motion and manipulation tasks in a collaborative way with the human.

We are conducting research in a multidisciplinary perspective, (1) running user studies and (2) developing an algorithmic framework able to integrate knowledge acquired through the trials. We illustrate here a first step by implementing a humanfriendly approach motion by the robot.
\end{abstract}

\section{INTRODUCTION}

The presence of humans in the robot environment and the necessity to interact with them raise a number of new questions and challenges. Clearly, the human should be taken explicitly into account in all steps of the robot design.

This paper addresses issues related to the close interaction between humans and robots from the standpoint of the motion decisions that must be taken by the robot in order to ensure:

- A safe motion, i.e., that cannot harm the human,

- A reliable and effective motion, i.e, that achieves the task adequately considering the motion capacities of the robot, and

- A user friendly motion, i.e, that takes into account a motion model of the human as well as his preferences and needs.

Let us consider a "simple fetch and carry task" as illustrated in figure 1 for a socially interactive robot [8]. The robot has to perform motion and manipulation actions and should be able to determine where a given task should be achieved, how to place itself relatively to a human, how to approach him, how to hand an object.

Our goal is to develop a robot that is able to take into account "social constraints" and to synthesize plans compatible

* The work described in this paper was partially conducted within the EU Integrated Project COGNIRON ("The Cognitive Companion") and funded by the European Commission Division FP6-IST Future and Emerging Technologies under Contract FP6-002020. with human preferences, acceptable by humans and easily legible in terms of intention.

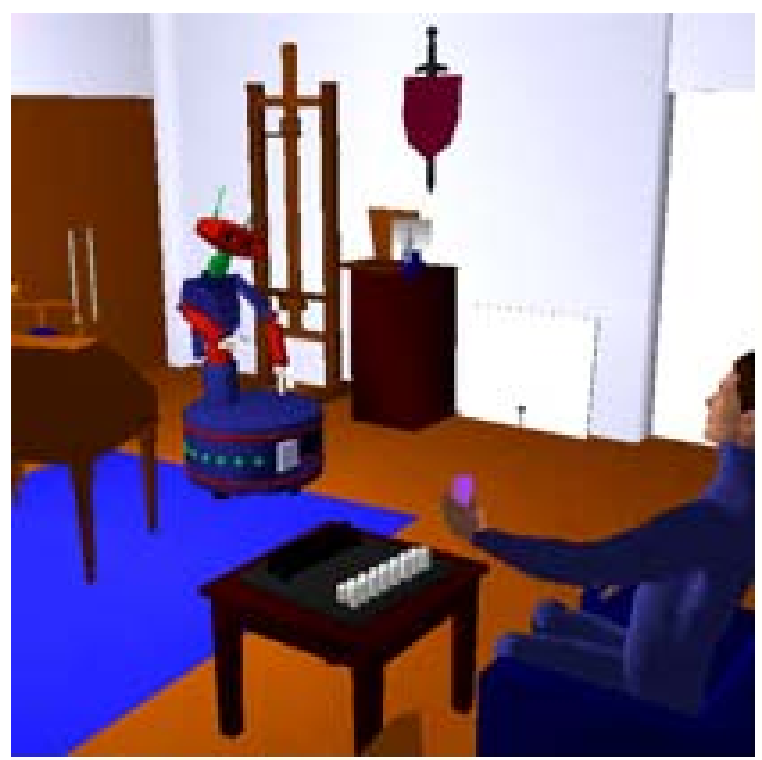

Fig. 1. A "fetch-and-carry" Scenario

All these questions have a particular flavour when the robot is a humanoid. Indeed, by its shape and functional abilities, it can act in very close interaction with humans, adopt postures and perform motions that are easily understandable by its human partners.

In this paper, we concentrate more precisely on navigation in the vicinity of humans. We are conducting research in a multidisciplinary perspective, (1) running user studies and (2) developing an algorithmic framework able to integrate knowledge acquired through the trials. We illustrate here a first step by implementing a human-friendly approach motion by the robot. Section II discusses related work. Section III presents trials that we have conducted in order to find out about subject preferences for the robot approach directions. Section IV presents the main characteristics of our navigation planner. Finally, we illustrate the outputs of a prototype implementation in section $\mathrm{V}$. 


\section{Human-Robot InTERACTION IN Literature}

A key issue is safety problems, where humans exist nearby robots. In industrial robotics, safety is assured by not allowing humans in a certain perimeter around robots and having emergency stop buttons [13]. Actually there is no interaction in these cases.

In a recent work by Nonaka et al. [18], the concept of safety has been studied by two aspects: "physical" safety and "mental" safety of human. Physical safety means that the robot do not physically injure humans. Mental safety, on the other hand, means that the motions of the robot do not cause any unpleasantness like fear, shock, surprise to human.

The physical safety is an absolute need for the human-robot interaction. It must be assured at the hardware and software design process of the robot. We can classify the safety strategies into two different types [11]: design strategies and control strategies. Besides new designs [3], [24] that will ensure safety at the physical level, fault-tolerant approaches [16] tend to detect and limit the consequences of hardware and software problems. A danger criterion is generally considered in control strategies and robot motions are executed by minimizing this criterion [14].

With these approaches physical safety is assured by avoiding collision with human and by minimizing the intensity of the impact in case of a collision. Another direction towards the motion in presence of humans is the research made for smart wheelchairs. Although there is not a real interaction between chair and human in a direct sense, the wheelchair motion needs to take into account the humans comfort [19].

In usual interactions between humans, some non written rules are respected that determine the distance between two persons (see the proxemic theory of E. T. Hall [10]). The robot should comply to similar conventions [4].

Other works try to imitate human motions for a better understanding of how humans behave in social environments. A recent work [2] makes robot place himself like humans in a conversation. We must note that this behaviour is only imitating humans self-placement.

Another approach that deals not only with safety but also implicitly comfort issues is the work on velocity profiles along a planned trajectory made by Alami et al. [1] where a robot adapts its trajectory and its speed to optimize the execution time and also to guarantee that no collision will occur. Although the human is not considered explicitly, this method guarantees a motion without collision by taking into account the sensor capabilities of the robot. Since the sensors have a certain range, it is likely necessary to slow down in some places of the robot's trajectory where the sensors are blocked by narrow passages or corners. And a velocity profile is found by optimizing the execution time.

While motion planning and control for humanoid robots deal with their specificities in terms of motion and manipulation [12], we claim that explicit reasoning on humans should also be integrated.

Although several authors propose motion planning or reac- tive schemes considering humans, there is no contribution that tackles globally the problem as we propose to do.

\section{HUMAN-ROBOT APPROACH DIRECTION TRIALS}

This section presents some relevant results from a demonstration Human-Robot Interaction trial event, which was run at the AISB Convention at the University of Hertfordshire (UH). The UH team are primarily interested in the human perspective of how robots could be useful in domestic environments; in particular the roles, tasks, and social behaviour that will be necessary for robots to exhibit in order to integrate into normal domestic situations.

In order to study human-robot relationships, they typically run HRI trials using carefully devised test scenarios, where the human centred view can be collected using a variety of methods. The "Wizard of Oz" (WOZ) technique, where robots are remotely controlled by human operators, is widely used in HRI studies where the human reactions to robot behaviour are investigated; see [22], [9], [17], [20]. The WOZ technique can quickly test proposed complex robot behaviours or capabilities. Subjects' opinions of social acceptability towards robot behaviour can be assessed before committing resources to develop fully autonomous capabilities, which may be expensive, technically difficult or even impossible to implement to date.

\section{A. The HRI Trial Method}

The chosen scenario involved a robot using different approach directions to bring a seated subject an object. The aims of the trial were to find out about subject preferences for the robot approach directions.

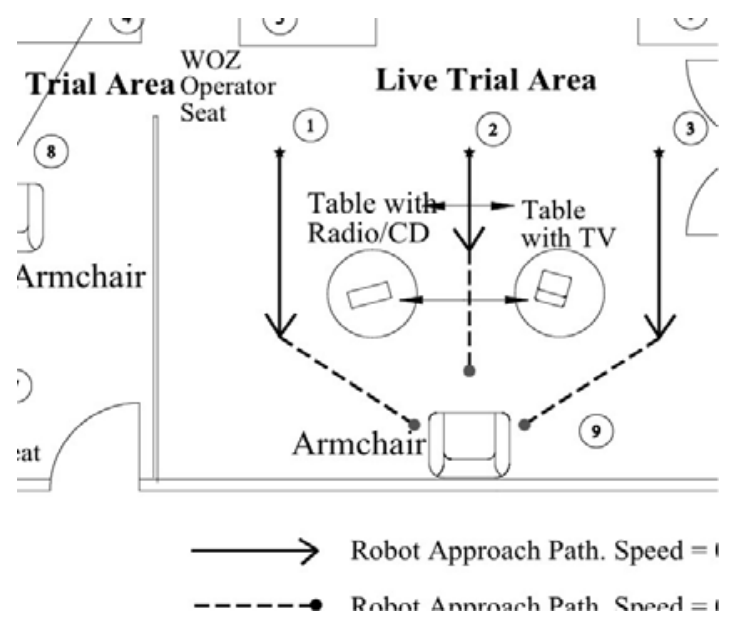

Fig. 2. Diagram of HRI approach trial experiment areas

The AISB demonstration event had an audience and was performed under non-laboratory conditions using 38 volunteers from the convention participants. There was also a follow up study carried out under more controlled conditions with 15 subjects and one of the aims of this trial was to verify and check results obtained from the demonstration study. The trial 
area was identically laid out for both trials to resemble a simulated living room with a chair and two tables. The subject was seated in the chair. To the left front and right front of the chair, two tables were arranged (with room for the robot to pass by) in front of the chair. One of the tables had a television placed upon it; the other had a radio and CD player. Posters were attached to the wall directly opposite the chair to provide a more comfortable atmosphere for the subject. The robot was driven to the appropriate start position by the WOZ operator. The robot's approaches to the subject were fully autonomous. The WOZ operator was seated at a table in the far corner of the room. Subjects were told that the robot would be controlled by the operator while it was driven to the three start positions, but would be approaching them autonomously to bring them the TV remote control. This was reinforced as the WOZ operator made notes and did not press any of the robot control keys (on the robot control laptop) while it approached the subject. (see Fig. 3 for example video clips).

\section{B. The HRI Trial Scenario}

The context was that the subject had arrived home, tired after a long day at work and rested in an armchair (Fig. 2). After looking around for the TV remote control, the subject then asked the robot to fetch it for them as they were too tired to get up. The robot then brought the remote control to the subject. It was explained to the subject that the robot was new to the household and it was necessary to find out which approach direction the subject preferred; either from the front (2), the left (1) or the right (3). The three possible paths taken by the robot are shown in Fig. 2. In order to justify the scenario of the robot fetching the remote control, one of the tables had a (switched off) TV set upon it. The other table had a CD-Radio unit. Our expectations prior to the trials were that subjects would prefer the approach from the front, since the robot was then fully visible at all times.
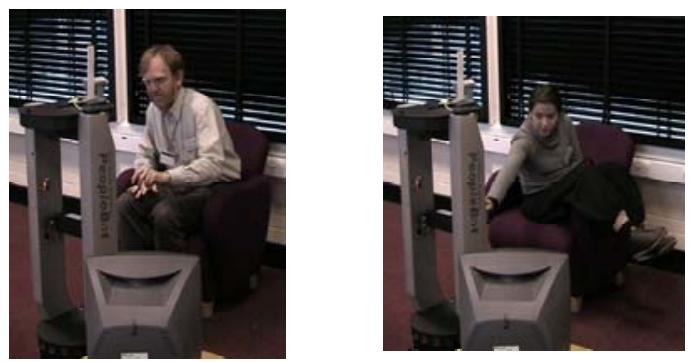

Fig. 3. Clips from the robot to human approach trials

A short introductory questionnaire was used to gain basic demographic and personal details from the subjects. At the end of each HRI trial a short questionnaire was used to assess the subjects' views on approach direction, approach speed, stopping distances, comfort levels and practicality for the different approach directions. The subjects' reactions to both live and video based HRI trials were also recorded by a single tripod mounted camera placed at an appropriate point, either (4), (5) or (6) (Fig. 2). Twenty one males (54\%) and 18 females (46\%) participated in the AISB robot approach direction trials. The mean age of subjects was 36 years (range: 22-58). Thirty five subjects (95\%) of subjects were right handed and 2 subjects $(5 \%)$ were left handed.

\section{Demonstration Trial Results}

a) Approach Direction Preferences: Fig. 4 illustrates that $60 \%(\mathrm{~N}: 23)$ of subjects stating preferred the right robot approach direction, followed by $24 \%(\mathrm{~N}: 9)$ preferring the left approach and just $16 \%$ (N: 6) preferring the front approach. An overriding majority of subjects stated least preferring the front robot approach direction (N: 31, 80\%). Few subjects least preferred the left and right approach directions.

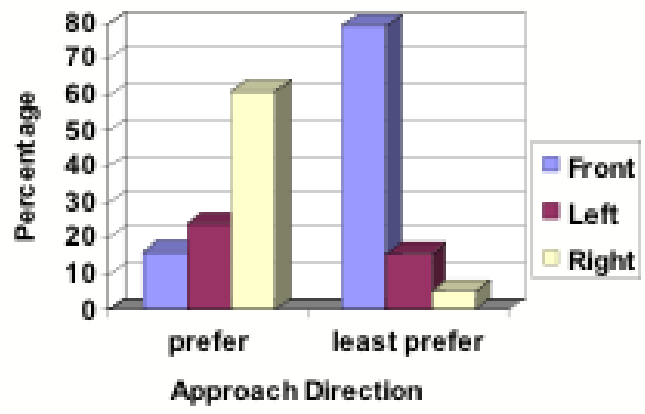

Fig. 4. Approach Directions Most and Least Preferred

Chi-square cross-tabulations revealed a non-significant association between gender and the robot approach direction preferred $(\mathrm{X} 2(2,38)=3.77, \mathrm{p}=0.1)$. More females stated that they preferred the robot front approach direction compared to males, and more males preferred the right robot approach direction compared to females (See Fig. 5).

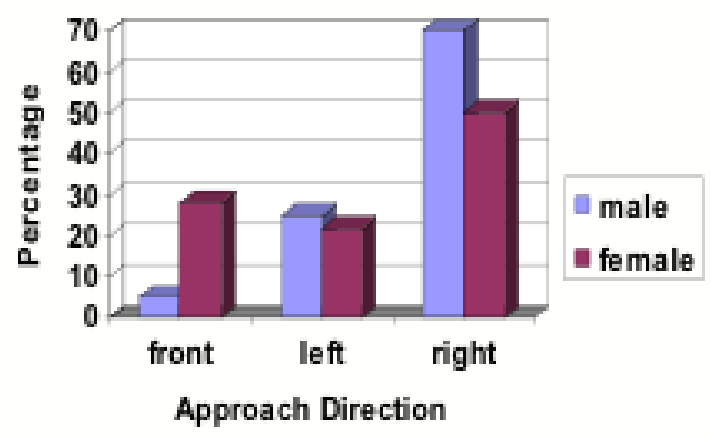

Fig. 5. Approach Direction Preferences by Gender 
A significant relationship was found between gender and least preferred robot approach direction $(\mathrm{X} 2(2,39)=7.09$, $p=0.03)$. Significantly more males stated least preferring the front robot approach direction compared to females (males: 95\%, females: 61\%). More females stated least preferring the right robot approach direction compared to males (males: $0 \%$, females: 11\%). Chi-square cross-tabulations revealed no significant relationships between age, handedness and approach directions preferred and least preferred.

b) Approach Distance Preferences: Subjects were asked to provide an overall rating (for all 3 approach directions) for the robot approach distance. $76 \%(\mathrm{~N}: 28)$ of subjects stated that the distance between them and the robot was 'about right', followed by $19 \%$ (N: 7) who felt that the robot was to 'too far' from them. Only $5 \%(\mathrm{~N}: 2)$ of subjects stated that the robot approached them too closely.

\section{HRI Trial Conclusions}

These results indicate that a large majority of subjects disliked robot approaching from the front approach direction. Some subjects sat with their legs over the side of the chair (see Fig. 2) and for these few cases, it may be that in this case a frontal approach (relative to the chair) was preferred. Thus there may be a more general rule that people do not like the robot to approach from the direction which their legs poke out. In the case of subjects who rated the front stopping distance as being too far, we observed that these subjects usually had their legs stretched out in front of them. This caused the robot to stop (due to the robot's stopping safety mechanism) when it reached the subject's feet, rather than moving close enough for them to reach the TV remote control comfortably.

\section{A Navigation Planner}

Today, the classical motion planning methods [15] are quite efficient to find feasible paths. However, the presence of humans in the environment drastically changes the notion of acceptable paths. In a human-robot interaction context, the computed paths do not only need to be collision-free but must also take into account the human comfort. This is illustrated on figure 6 which shows two paths possibly produced by a classical motion planner. Obviously, both paths are badly chosen since one path passes too close to the wall, causing a surprise to human, and the other passes behind of the human also causing some discomfort.

The User studies with humans and robots reported in the previous section (see also [23][2]) provide a number of properties required when dealing with humans. Only very limited works consider such comfort and legibility issues, often in an ad hoc manner. We describe below a new technique that allows to integrate such additional constraints in a more generic way. First, we introduce three criteria to the motion planning stage to ensure the safety and comfort. The robot must take into account these three criteria at the planning stage along with

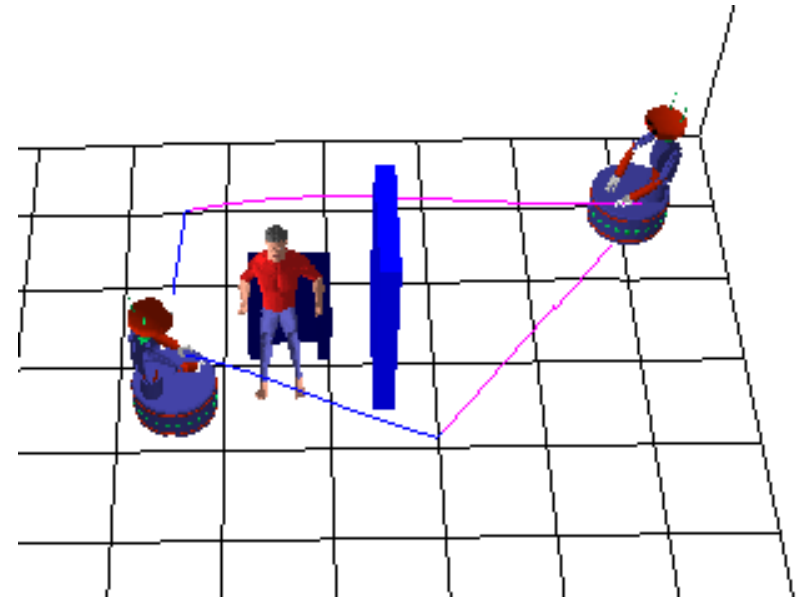

Fig. 6. Two paths found by classical motion planning

the more common aspects of path planning such as obstacle avoidance and shortest path finding.

Each criterion is represented by a numerical potential stored in a $2 \mathrm{D}$ grid combining various costs. These costs are highly related to the humans' state, capabilities and preferences. The grid $G$ can be defined as follows:

$$
G=\left(M_{n, p}, H_{1} \ldots H_{n}\right)
$$

where $M_{n, p}$ is a matrix containing $n * p$ cells represented by , $a_{x, y}$, the cost of the coordinate $(i, j)$ in a $2 \mathrm{D}$ plane and $H_{1} \ldots H_{n}$ is a list of humans in the environment. A human $H_{i}$ is modeled by $H_{i}=\left(\right.$ St, State St $_{1} \ldots$ State $\left._{n}\right)$ where $S t$ is the structure and kinematics of the human and State $_{i}$ is a human state defined by a number of cost parameters and state description:

$$
\text { State }_{i}=(\text { Name, Conf }, \text { Param })
$$

where Name is the name of the state (for ex. Name = SITTING, STANDING), Conf is the humans configuration in that state and Param represents the data needed to compute costs according to that state.

From the user studies above, we extracted three criteria that will allow the robot to be more human friendly.

\section{A. Safety Criterion}

The first criterion, called safety criterion, mainly focuses on ensuring the safety by controlling the distance between robot and human. The robot, if possible, must avoid approaching too much to human, and in some cases a certain perimeter around human must not be allowed to pass through. However, the robot must be able to approach the human because of the necessity of their interaction (for example to handle some object to the human). Hence, this distance between the robot and the human is not uniform and fixed, but depends on the interaction. The feeling of security is highly dependent to the humans personality and physical capabilities. For example, a robotics scientist feels much comfortable when a robot is 
around than an other person who sees the robot first time. Also, an elderly person can feel safer when the robot is 4 meters away from him than a curious teenager who feels safe even when the robot approaches very close. The humans current state plays also an important role as the safety feeling differs highly when the human is sitting than when he is standing up. When the human is sitting, as his mobility is reduced, he tends to have a low tolerance to the robot getting close. On the contrary when standing up he gets a higher mobility, therefore allowing the robot to come closer.

The user studies on the spatial interaction between people indicate that the spaces 1-3 meters away from humans are considered as interaction spaces with non-friends and $>3 \mathrm{~m}$ zones are considered as public zones. These studies can be used to define the distance limitations between robots and humans [10], [5], along with the studies of social spaces between robots and humans [23]

The safety grid contains a human centered gaussian form of cost distribution. Each coordinate $(x, y)$ in this grid contains a cost inversely proportional to the distance to the human. Then, when the distance between the human and a point in the environment (in the grid) $D\left(\left(x_{i}, y_{j}\right)\right)$ is greater than the distance of another point $D\left(\left(x_{k}, y_{l}\right)\right)$, we have $\operatorname{Cost}\left(x_{k}, y_{l}\right)>$ $\operatorname{Cost}\left(x_{i}, y_{j}\right)$. Since the safety concerns loose their importance when the robot is far away from the human, the cost also decreases when getting farther from the human, until some maximal distance at which it becomes null.

Figure 7 shows a computed safety grid attached to a human who is sitting on a chair. The vertical lines represent the cost associated to each cell. As shown by the figure, the cost is maximal at the human position and the cost range considered for a sitting human is approximately 3 meters ${ }^{1}$.

As mentioned above, this cost highly depends on the human state and figure 8 shows a different cost computed for a standing human. As his mobility increases, the cost influence range shrinks to approximately 2 meters .

Other types of human state can be easily taken into account. For example, costs associated to states like sleeping, awake, ... can be easily handled with corresponding Params.

Once the grid is computed, searching for a minimum cost path will avoid to move too close to the human since approaching the human is more costly than staying far away.

\section{B. Visibility Criterion}

The human comfort is another criterion to be considered when dealing with human-robot interactions. In particular, the human generally feels more comfortable when he sees the robot. Therefore, we introduce another criterion, called visibility criterion, in order to help the robot to stay in the field of view of the human during its motions.

\footnotetext{
${ }^{1}$ These values are only estimates and can be changed according to the context.
}

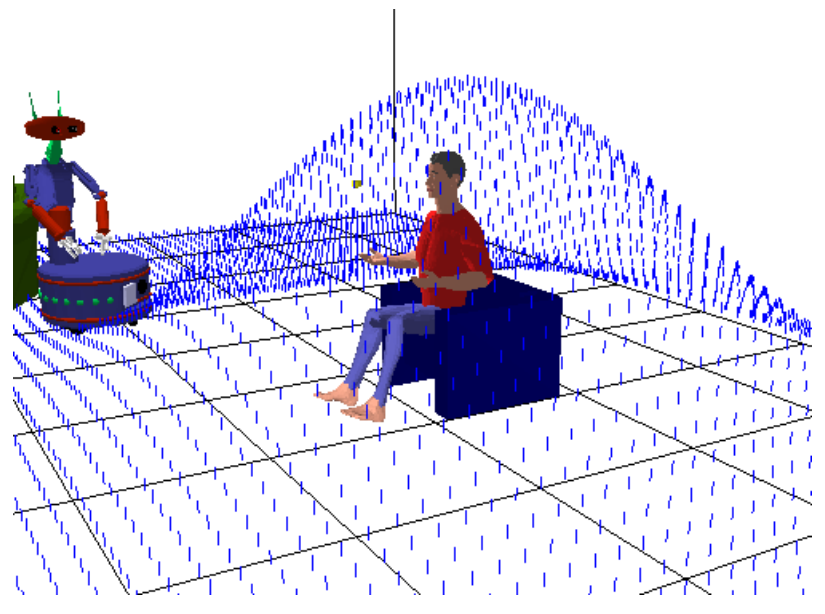

Fig. 7. Safety grid when human is sitting

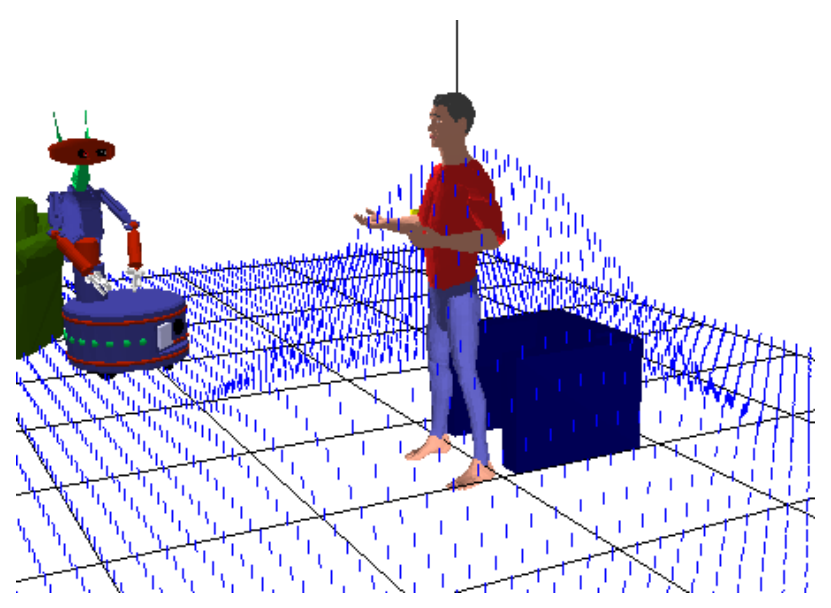

Fig. 8. Safety grid when human is standing

The visibility grid is constructed according to costs reflecting the effort required by the human to get the robot in his field of view. Grid points located in a direction for which the human only has to move his eyes have a lower cost than positions requiring to move the head in order to get the robot in the field of view. Also, when the robot is far away from the human, the effect of the visibility must decrease. The computed visibility costs are shown on figure 9 . The zone situated in front of the human has very low costs. On the contrary, the zone situated behind the human has higher costs. As the visibility has a certain range of effect, the intensity decreases according to the distance to the human and its effect becomes negligible after 3-4 meters. Also, since the grid is attached to the head of the human, the computed costs are actualized when the human changes his field of view (turn his head) in planning and/or execution stage.

\section{Hidden Zones}

In the grids illustrated above, the costs are calculated without taking into account the obstacles in the environment. However, 


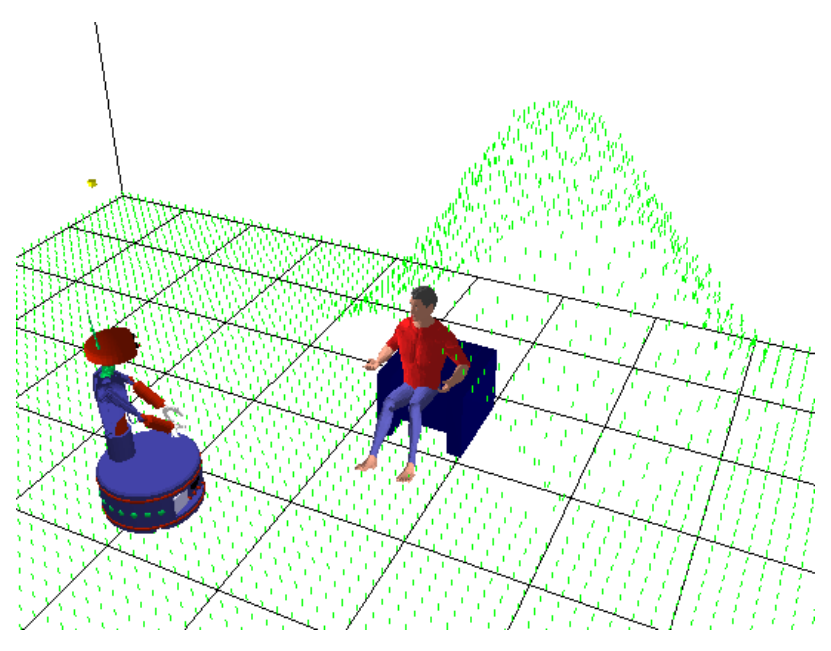

Fig. 9. Visibility grid

obstacles in the close vicinity of the human can have various effects on the safety and comfort. If the robot is behind an obstacle, the human would feel much comfortable because the obstacle would block the direct way between human and the robot. So the distance criterion must be cancelled in the zones located behind the obstacles.

On the other hand, as the robot becomes hidden when it passes behind an obstacle, the visibility costs do not correspond anymore to physical realities. To handle this issue, we introduce another criterion additional to visibility and safety, called "hidden zones" criterion. This criterion helps to determine better costs for positions hidden by the obstacles.

Hence, an important effect of obstacles to the comfort of the human is the surprise factor. When the robot is hidden by an obstacle close to the human and suddenly appears in the human field of view, it can cause surprise and fear. To avoid this effect, we must discourage the robot to pass behind an obstacle too closely, and must allow it to get into the humans field of view when sufficiently far from the human. This can be done by putting costs to the zones hidden from the view by the obstacles.

The costs in the hidden zone grid is inversely proportional to the distance between the human and the robot. The range of the effect of the surprise factor is approximately $3 \mathrm{~m}$, so the costs decrease to zero in the $3 \mathrm{~m}$ perimeter and remains null for the other grid points (Fig. 10).

\section{Path planner}

Once the safety, visibility and hidden zones grids have been computed, they are merged to one single grid that the robot will search for a minimum cost path. Note that we do not compute the four grids ( 3 criteria +1 final) explicitly but just the costs of the grid cells necessary during search. Different ways can be used to merge the grid costs. A first way can be to compute the overall cost from the weighted sum of the elementary costs

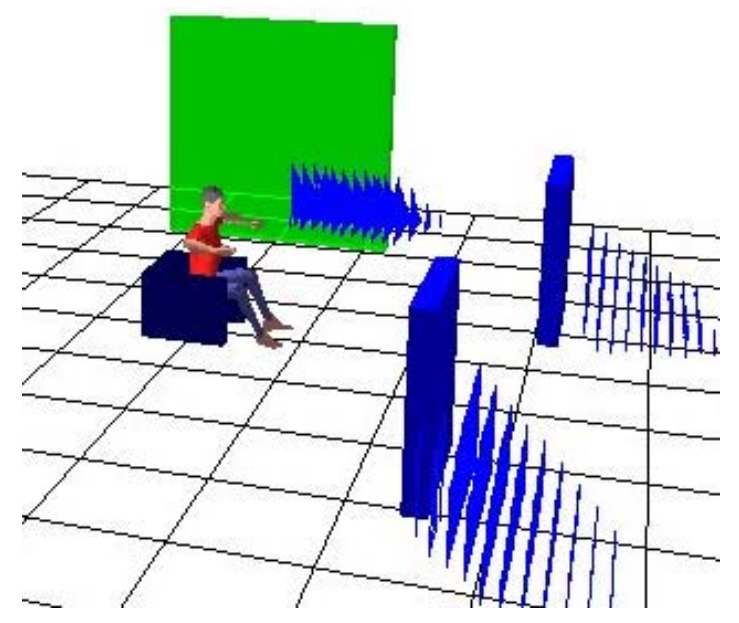

Fig. 10. Decreasing costs of hidden zones grid

$\operatorname{Cost}_{\text {merged }}(x, y)=w_{1} \operatorname{Cost}_{\text {safety }}(x, y)+w_{2} \operatorname{Cost}_{\text {visibility }}(x, y)$

where $(x, y)$ is a grid point, $w_{1}$ is the weight of the safety grid and $w_{2}$ is the weight of the visibility grid.

Another way can be to consider the maximum cost values when merging the grids

$\operatorname{Cost}_{\text {merged }}(x, y)=\max \left(\operatorname{Cost}_{\text {safety }}(x, y), \operatorname{Cost}_{\text {visibility }}(x, y)\right)$

Note that we do not merge hidden zones grid with the other 2 grids. That is mainly because hidden zones grids serves as a replacement of this 2 grids for positions where the robot could be seen if it wasn't blocked by an obstacle. The final grid is computed by:

if ( $R$ is on $(x, y)$ AND

$R$ is in field of view of $H_{i}$ AND

$H_{i}$ cannot see $R$ because of an obstacle $O$ )

then $\operatorname{Cost}_{\text {final }}(x, y)<-w_{3} \operatorname{Cost}_{\text {hiddenzones }}(x, y)$

else $\operatorname{Cost}_{\text {final }}(x, y)<-\operatorname{Cost}_{\text {merged }}(x, y)$

Our planner can use both ways depending on the task and on the balance between criteria. For example, for an urgent task, the importance of the visibility grid is less than the safety grid so that the robot does not take to much into account the visibility.

Once the final grid is computed, the cells corresponding to the obstacles in the environment are labeled as forbidden and an $\mathrm{A}^{*}$ search is performed to find minimum-cost path between given two positions of the robot. The computed path is collision-free and also takes into account the human comfort and safety.

\section{Results}

The navigation planner is implemented ${ }^{2}$ within the Move3D [21] software platform developed at LAAS.

${ }^{2} \mathrm{~A}$ number of mpeg animations of computed motions can be found at http://www.laas.fr/ easisbot 
Figure 11 shows a first path computed in the presence of two humans looking each other. One can note that the computed solution corresponds to a direct path remaining at equal distance from both humans. Consider now the same problem solved in a situation where one of both humans is turned back. Even the previous path is feasible, the planner chooses a more comfortable path along which the robot remains visible for both humans (figure 12).

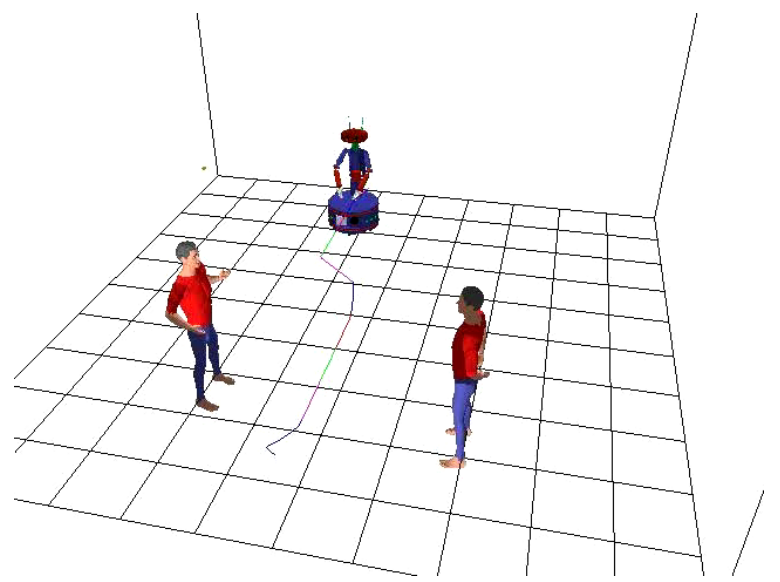

Fig. 11. The robot chooses a path with maximum safety and comfort

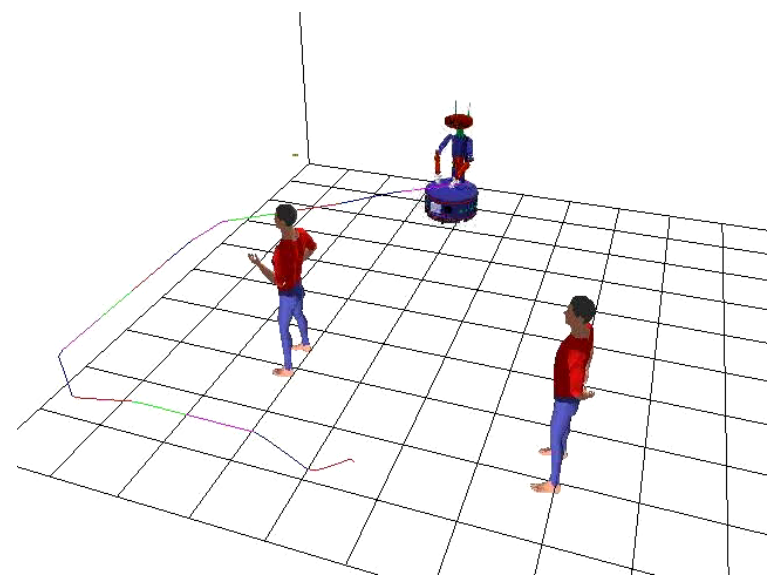

Fig. 12. The path changes according to the visibility grids

Figure 13 illustrates another scenario with two humans sitting in a room. The robot is initially located in the right corner of the room and has to move next to the human hidden by the wall obstacle. The figure shows the safety and visibility grids computed for each human, and the hidden zone created by the wall.

The minimum cost path computed by the planner (figure 14) has the following characteristics:

- The robot does not approach too close to both humans. It chooses a solution that only enters in the humans $3 \mathrm{~m}$ zone in the last portion of the path.

- The robot remains as visible as possible along its path. Because of the hidden start position, there is no possibility

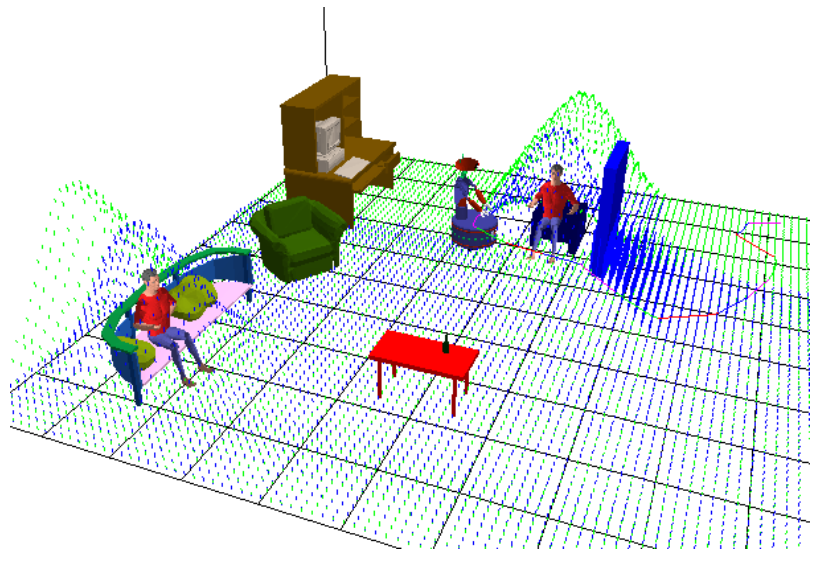

Fig. 13. The 3 types of grids

to be in the human field of view at the beginning of path. Therefore the planner chooses to pass behind the wall instead of passing behind the human.

- The robot is not too close to the human when it appears in its field of view. The transition from the hidden zone behind the wall to the visible one is sufficiently far from the human to avoid any surprise effect. Then the robot can approach to the human to reach its final position.

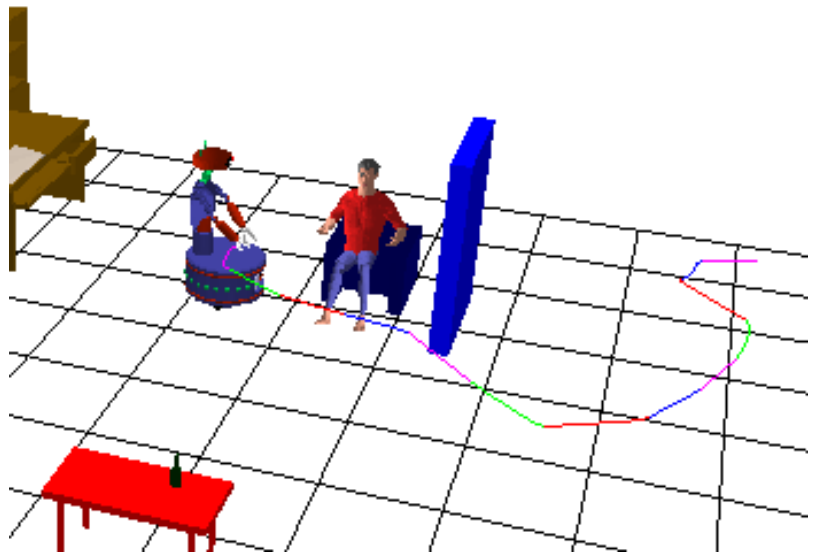

Fig. 14. The computed path takes into account the safety and the comfort of the human

As in real world scenarios there can be multiple objects and humans in motion, if the environment changes during execution, the calculated path can loose its validity. This trajectory can be replaced very rapidly by replanning that invalid trajectory. As HAMP's planning method is fast (the trajectories for both examples are produced in less than a second with and AMD Athlon 1.8 Mobile processor), replanning allows us to be sufficiently reactive to the environment changes and to execute trajectories smoothly making the replanning totally transparent to the humans. . 


\section{CONCLusion And Future Work}

We have proposed a navigation planner that takes into account the humans existence explicitly and that not only elaborates safe robot paths, but also plans good, socially acceptable and legible paths.

It is based on a multidisciplinary perspective. The algorithmic framework that we propose is able to integrate results provided by user studies.

This is a small step towards a very ambitious goal. Indeed, there a already a number of extensions that can be envisaged. For instance, it is clearly necessary to consider speed and acceleration and their influence on human comfort and acceptability of the robot actions. Besides, it remains to implement such schemes on real robot and to conduct validation tests.

We are also planning to develop a manipulation planner in order to allow the robot to hand objects to a human while respecting the safety and social constraints.

\section{REFERENCES}

[1] R. Alami, T. Simeon and K. Madhava Krishna, "On the influence of sensor capacities and environment dynamics onto collision-free motion plans", Proc. IEEE Int. Conf. on Intelligent Robots and Systems, 2002.

[2] P. Althaus, H. Ishiguro, T. Kanda, T. Miyashita and H. I. Christensen, "Navigation for human-robot interaction tasks", Proc. in IEEE Int. Conf. on Robotics \& Automation, New Orleans, USA, 2004.

[3] A. Bicchi and G. Tonietti, "Fast and Soft Arm Tactics: Dealing with the Safety-Performance Trade-Off in Robot Arms Design and Control", IEEE Robotics and Automation Magazine, IEEE Volume 11, Issue 2, June 2004 Page(s):22 - 33

[4] R. Chatila , R. Alami , T. Simeon , J. Pettre , L. Jaillet, "Safe, reliable and friendly interaction between humans and humanoids", Rapport LAAS No02530, 3rd 3rd IARP International Workshop on Humanoid and Human Friendly Robotics, Tsukuba (Japan), 11-12 December 2002, pp.83-87

[5] H. I. Christensen and E Pacchierotti, "Embodied social interaction for robots", Proc. AISB'05 Symposium on Robot Companions: Hard Problems and Open Challenges in Human-Robot Interaction, 2005, pp.40-45.

[6] A. Clodic, V. Montreuil, R. Alami, R. Chatila, "A Decisional Framework for Autonomous Robots Interacting with Humans" Proc. IEEE Ro-man 2005, Nashville, August 2005.

[7] Cogniron Project Web Site, "http://www.cogniron.org"

[8] T. Fong, I. Nourbakhsh, and K. Dautenhahn, "A survey of socially interactive robots," Robotics and Autonomous Systems, vol. 42, pp. $143-$ 166.

[9] A. Green, H. Huttenrauch and K Severinson Eklundh, "Applying the Wizard of Oz Framework to Cooperative Service discovery and Configuration," Proc. IEEE Ro-man 2004, 13th IEEE International Workshop on Robot and Human Interactive, Oka-yama, Japan, IEEE Press, 2004.

[10] E. T. Hall, "The Hidden Dimension”, Doubleday, Garden City, N.Y., 1966.

[11] K. Ikuta, H. Ishii and M. Nokata, "Safety evaluation methods of design and control for human-care robots", The International Journal of Robotics Research, Vol 22, No. 5, 2003, pp. 281-297.

[12] J.J. Kuffner, K. Nishiwaki, S. Kagami, M. Inaba, and H. Inoue. "Motion planning for humanoid robots". In Proc. 20th Int'l Symp. Robotics Research (ISRR'03), Italy, October 2003.

[13] D. Kulic and E. Croft, "Strategies for safety in human-robot interaction", Proc. IEEE Int. Conf. on Advanced Robotics, 2003, pp. 810-815.

[14] D. Kulic and E. Croft, "Safe planning for human-robot interaction," Proc. in IEEE Int. Conf. on Robotics \& Automation, New Orleans, USA, 2004.

[15] J.C. Latombe, Robot Motion Planning, Kluwer Academic Publishers, Boston, MA, 1991.

[16] B. Lussier, A. Lampe, R. Chatila, J. Guiochet, F. Ingrand, M.O. Killijian, D. Powell, "Fault tolerance in autonomous systems: how and how much?", 4th IARP/IEEE-RAS/EURON Workshop on Technical Challenges for Dependable Robots in Human Environments, Nagoya (Japan), 16-18 Juin 2005, 10p.
[17] D. Maulsby, S. Greenberg and R Mander, "Prototyping an intelligent agent through Wizard of Oz," Proc. ACM SIGCHI Conference on Human Factors in Computing Systems, Amsterdam, The Netherlands, ACM Press, pp. 277-284, 1993.

[18] S. Nonaka, K. Inoue, T. Arai and Y. Mae, "Evaluation of human sense of security for coexisting robots using virtual reality", in. Proc IEEE Int. Conf. on Robotics \& Automation, New Orleans, USA, 2004.

[19] R. S. Rao, K. Conn, S. H. Jung, J. Katupitiya, T. Kientz, V. Kumar, J. Ostrowski, S. Patel and C. J. Taylor, "Human-robot interaction: Application to smart wheelchairs," Proc. in IEEE Int. Conf. on Robotics \& Automation, Washington, DC, 2002.

[20] B. Robins, K. Dautenhahn and J. Dubowski, "Investigating Autistic Children's Attitudes Towards Strangers with the Theatrical Robot - A New Experimental Paradigm in Human-Robot Interaction Studies," Proc. 13th IEEE International Workshop on Robot and Human Interactive. Okayama, Japan, IEEE Press, pp. 557-562, 2004.

[21] T. Siméon, JP. Laumond, F. Lamiraux. Move3D: a generic platform for motion planning. 4th International Symposium on Assembly and Task Planning, Japan, 2001.

[22] M. Walters, K. L. Koay and K. Dautenhahn, "Practical and Methodological Challenges in Designing and Conducting Interaction Studies with Human Subjects," Robot Companion Symposium at the. Convention on Social Intelligence and Interaction in Animals, Robots and Agents. (AISB'05). 12th - 15th April 2005. University of Hertfordshire, UK.

[23] M.L. Walters, K. Dautenhahn, R. te Boekhorst, K. L. Koay, C. Kaouri, S. Woods, C. Nehaniv, D. Lee, I. Werry , "The Influence of Subjects Personality Traits on Personal Spatial Zones in a Human-Robot Interaction Experiment", Proc. IEEE Ro-man 2005, Nashville, August 2005.

[24] M. Zinn, O. Khatib, B. Roth, J.K. Salisbury, "Playing it safe [humanfriendly robots]", Robotics \& Automation Magazine, IEEE Volume 11, Issue 2, June 2004 Page(s):12 - 21 